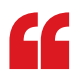

site-specific recombination of $h s d S$ and the associated changes in HsdM methylation patterns constitute an epigenetic switch that controls colony morphology

BACTERIAL Genetics

\title{
SMRT-seq reveals an epigenetic switch
}

Streptococcus pneumoniae uses genetic diversification as a strategy to achieve phenotypic plasticity. For example, DNA inversion of the $h s d S$ genes of type I restriction-modification (R-M) systems determines whether S. pneumoniae forms opaque or transparent colonies, which have different colonization and virulence characteristics. Zhang and colleagues now use single-molecule, real-time sequencing (SMRT-seq) to show the allelic variation of $h s d S$ that results from site-specific recombination forms part of an epigenetic switch.

The long sequencing reads that are generated by SMRT-seq enabled a characterization of DNA rearrangements in a multidrug-resistant strain of S. pneumoniae. Nine configurations of $h s d S$ were identified, each of which was the product of site-specific DNA recombination events. As HsdS confers sequence specificity to an endonuclease (HsdR) and a methyltransferase (HsdM), the authors hypothesized that structural variation of $h s d S$ might modulate the functionality and/or sequence specificty of HsdS, thus altering the DNA methylation pattern produced by HsdM. Indeed, SMRT-seq, which is notable for its ability to generate single base-resolution methylome data, showed that phase variation of $h s d S$ resulted in either a loss of functionality (three alleles) or a

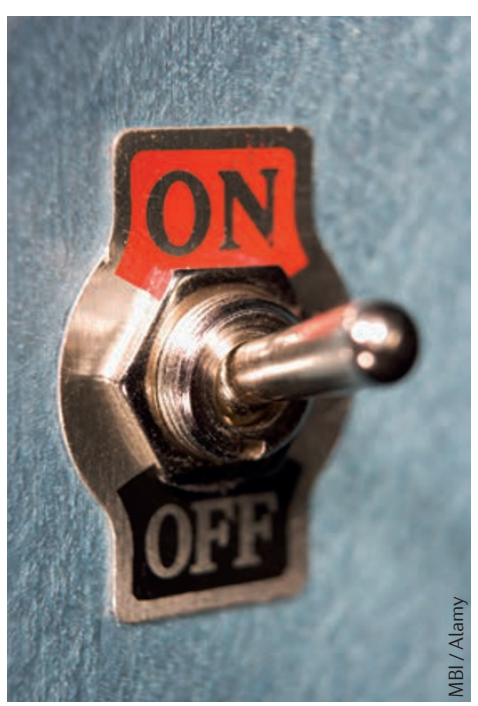

variation in the methylation motif (six alleles). Furthermore, each allele was uniquely associated with either opaque or transparent colonies, which corresponded to variable adhesion and colonization abilities.

Genetic experiments confirmed that the methyltransferase activity of HsdM was required for switching between the two phenotypes, leading the authors to conclude that site-specific recombination of $h s d S$ and the associated changes in HsdM methylation patterns constitute an epigenetic switch that controls colony morphology. As DNA rearrangements of $h s d S$ are present in many bacterial species, the ability to operate as an epigenetic switch may be a common function of this gene, which would represent an intriguing departure from the well-characterized roles of R-M systems in genome defence.

Naomi Attar

ORIGINAL ARTICLE Li, J. et al. Epigenetic switch driven by DNA inversions dictates phase variation in Streptococcus pneumoniae. PLoS Pathog. 12, e1005762 (2016) 